\title{
Single Pick Cutting Rock Load Identification Based on Improved Regularization Method
}

\author{
Lei Dong, ${ }^{1,2}$ Siyu Zhai, ${ }^{1}$ Bukang Wang, ${ }^{2,3}$ Liang Dong, ${ }^{2,3}$ Junyuan Wang, ${ }^{1}$ and Ruimin Shi $\mathbb{I D}^{1}$ \\ ${ }^{1}$ School of Mechanical Engineering, North University of China, Taiyuan 030051, China \\ ${ }^{2}$ National Engineering Laboratory of Coal Mining and Excavation Machinery Equipment, Taiyuan 030032, China \\ ${ }^{3}$ Taiyuan Institution, China Coal Technology and Engineering Group, Taiyuan 030006, China \\ Correspondence should be addressed to Ruimin Shi; 20150019@nuc.edu.cn
}

Received 1 April 2021; Revised 17 May 2021; Accepted 5 July 2021; Published 13 July 2021

Academic Editor: Fidelis Tawiah Suorineni

Copyright () 2021 Lei Dong et al. This is an open access article distributed under the Creative Commons Attribution License, which permits unrestricted use, distribution, and reproduction in any medium, provided the original work is properly cited.

To explore the relationship between the cutting vibration and the cutting load of a single pick, this paper studied a new method for a single pick cutting rock load identification. This paper improved the low accuracy problem of the regularization method in the inverse process of frequency response function in the traditional load identification method by introducing a filter operator. By combining the inverse pseudoexcitation method and the improved regularization method, the identification of the load dependent on the vibration signal was realized. A single pick cutting rock test equipment was built, which could simulate the actual working conditions of pick cutting rock in the underground or tunnel. By changing cutting speed, cutting angle, cutting spacing, and cutting depth of the single pick, the change trends of real cutting load and identification load were obtained. The load identification method proposed in this paper was consistent with the change trend of the real load under the single pick cutting state. Therefore, the method had good recognition accuracy and the maximum load recognition error was $17.35 \%$. Compared with other traditional load identification methods, the identification error was reduced by a maximum of $1.98 \%$. This method can identify the cutting load of single pick and modify the morbidity problem of frequency response function matrix. The method has a better recognition effect on the cutting load of the pick than the traditional recognition methods. The research could benefit the design of the cutting system and the arrangement of the pick on the coal mine or tunneling machinery.

\section{Introduction}

The cutting system is the most important mechanical system in coal mining or tunneling machinery. It is the key to determine the cutting performance and cutting efficiency. The pick is the part of the cutting system that is in direct contact with the cutting object, which bears the cutting load and completes the tasks of rock entering and rock breaking. Parameters such as cutting spacing, cutting speed, cutting angle, and cutting depth of the pick according to the arrangement and combination of the pick play an essential role in the design and manufacture of the cutting system. Therefore, one of the current research topics of tunneling machinery is studying the effect of cutting parameters on rock breaking load based on hardness and other material properties of the cutting tool.

Acquiring the cutting load generally adopts the method of directly measuring the cutting power. However, the cutting power is often mixed with auxiliary power such as walking power, hydraulic power, and dust removal power, which are not easy to distinguish. The cutting power also contains comprehensive cutting information such as the feed force and the rotation force, which make it difficult to obtain the information of the pick breaking load [1]. Therefore, it is necessary to build a single pick rock breaking test system and to establish a method to study the influence of a single pick cutting parameters on the cutting load. Dogruoz and Bolukbasi [2] used picks with different degrees of wear and conducted cutting tests on a variety of different 
types of rocks. They obtained the law of the single pick cutting energy. Yang et al. [3] conducted cutting experiments on picks with different cutting angles and studied the influence of pick installation parameters on pick wear.

Load identification is the inverse problem of structural dynamics. It is a process of identifying excitations according to characteristic parameters and responses of the structure itself. Load identification technology has been widely used in various fields such as structural health monitoring in civil engineering and durability testing in the automotive field $[4,5]$. Load identification methods can be roughly divided into three categories: time domain, frequency domain, and modern intelligence [6-8]. Time domain method includes series expansion method, Kalman filter method, and inverse system method [9-11]. The application in complex mechanical systems has great limitations, because the inputs and outputs of the time domain system are relatively complicated convolution relationship and the amount of calculation after discretization is too large. Modern intelligence method includes neural network method, wavelet transform method, and genetic algorithm method [12-16]. It is rarely used in practical engineering applications, because the modern intelligence algorithm requires a large number of training samples and the establishment of the topology structure is difficult [17]. Although the frequency domain method also has many shortcomings such as morbidity of frequency response function and modal truncation, it is widely used in actual engineering situations because it requires fewer training samples and the calculation amount is small.

The frequency response matrix in the load inversion problem is often morbid. The proper handling of the morbidity problem is the key to the success of load identification. Choi et al. $[18,19]$ used Tikhonov regularization method to improve the stability of load identification results and compared the effect of different regularization parameter selection methods on load identification accuracy.

In this paper, the inverse pseudoexcitation method in the frequency domain method is used to identify the load of a single pick rock breaking load. The modified regularization method is used to solve the morbidity problem encountered in the inverse process of the frequency response function matrix. A single pick rock breaking test equipment was built to obtain the trend of the pick cutting load with the cutting angle, the cutting speed, the cutting spacing, and the cutting depth. Finally, the identification load is compared with the actual measured value and the reason for the error is analyzed. The accuracy of the load identification before and after the method improvement is also analyzed. The test results show that the modified regularization method can improve the accuracy of single pick cutting load identification. The research method can lay a theoretical foundation for the design of the cutting system of the tunneling machinery and the improvement of cutting efficiency.

\section{Load Identification Method}

2.1. Inverse Pseudoexcitation Method. One of the most used methods in load identification is the inverse pseudoexcitation method [20]. The motion equation of structure under random excitation is expressed as

$$
[M]\{\ddot{x}\}+[C]\{\dot{x}\}+[K]\{x\}=\{F\},
$$

where $[M],[C]$, and $[K]$ represent $n \times n$ orders of mass matrix, damping matrix, and stiffness matrix, respectively; $\{F\}$ represents the $n$-dimensional external force vector. The conversion formula of the power spectral density matrix is expressed as follows:

$$
\left[S_{Y Y}\right]=[H]^{*}\left[S_{F F}\right][H]^{T} .
$$

In equation (2), $\left[S_{Y Y}\right]$ is $l \times l$ order response power spectral density; $[H]$ is $l \times m$ order frequency response function matrix; $l$ and $m$ are the numbers of degrees of freedom of response and excitation, respectively, and $l \geq m$; the superscripts $*$ and $T$ represent complex conjugate and transpose of the matrix, respectively. Both sides of equation (2) are multiplied by the inverse matrix of the frequency response function matrix to obtain

$$
\left[S_{F F}\right]=[H]^{+*}\left[S_{Y Y}\right][H]^{+T} .
$$

In equation (3), $\left[S_{F F}\right]$ is $m \times m$ order excitation power spectral density matrix; "+" means seeking generalized inverse. The frequency response function $[H]$ is generally not a square matrix but a general form of direct inversion, which is a very computationally intensive task for complex structures. Seeking the generalized inverse directly is often ineffective.

For the inverse problem of multipoint $(l>m)$ arbitrary excitation, the known response spectrum matrix is decomposed into

$$
\left[S_{Y Y}\right]=\sum_{j=1}^{r}\{b\}_{j}^{*}\{b\}_{j}^{T},
$$

where $r$ is the rank of $\left[S_{Y Y}\right]$ and $m \leq r ;\{b\}_{j}$ is the $j$-th order feature pair of the Hermite matrix. To construct a pseudoresponse,

$$
\{\tilde{y}\}_{j}=\{b\}_{j} e^{i \omega t} .
$$

To get inversion corresponding pseudoexcitation,

$$
\begin{aligned}
\{\tilde{f}\}_{j} & =[H]^{+}\{\tilde{y}\}_{j} \\
& =[H]^{+}\{b\}_{j} e^{i \omega t}\{a\}_{j} e^{i \omega t} .
\end{aligned}
$$

Therefore, the excitation spectrum matrix can be obtained as

$$
\left[S_{F F}\right]=\sum_{j=1}^{r}\{\widetilde{a}\}_{j}^{*} \cdot\{\widetilde{a}\}_{j}^{T},
$$

where $\{\tilde{a}\}_{j}=[H]^{+}\{b\}_{j},\left\{b_{j}\right\}=\sqrt{\lambda_{j}} \cdot\left\{\psi_{j}\right\}^{*}, \lambda$ is the eigenvalue of $\left[S_{Y Y}\right]$, and $\psi$ is the eigenvector.

The frequency response function of the system can be obtained by the finite element method. The test device is a single pick and single swing device; hence, it can be simplified to a cantilever beam. The free mode and working mode of the cantilever beam could be obtained in [21, 22]. Therefore, the solution of the natural frequency response 
function of the test device simplified as a cantilever beam will not be explained in detail in this paper.

2.2. Modified Regularization Method. The inverse pseudoexcitation method needs to invert the frequency response function to solve the pseudoexcitation or test excitation. The regularization method to solve the inverse matrix of the frequency response function is a method that is easy to understand and has a short calculation time. However, this method often results in a lower load identification accuracy because of morbidity matrix or improper selection of parameter values. Therefore, this paper established a new selection criterion for the key parameters in the regularization method to improve the accuracy of the traditional load identification method.

2.2.1. Singular Value Decomposition. The singular value decomposition method is often used to calculate the generalized inverse of the matrix. The singular value decomposition of $\mathrm{H}$ is as follows:

$$
[H]=[U][S][V],
$$

where $[U]$ represents the left singular value vector; $[V]$ represents the right singular value vector; and $[S]$ represents the singular value vector.

Since the matrix is morbid at the natural frequency during the inversion, in general, the frequency response function inverse matrix $[\mathrm{H}]^{+}$can be expressed as

$$
[H]^{+}=\sum_{i=1}^{m} \frac{1}{S i} u_{i}^{T} v_{i},
$$

where $\mathrm{H}^{+}$is Moore-Penrose generalized inverse; $u_{i}$ represents the left singular value vector; $v_{i}$ represents the right singular value vector; and $s_{i}$ represents the singular value.

2.2.2. Modified Regularization Method. If (1) the singular value of matrix $H$ gradually becomes zero and (2) the condition number of matrix $H$ is too large, that is, the ratio between the largest singular value and the smallest singular value of structure matrix $H$ is larger, when one of the above conditions is met or all conditions are met at the same time, the problem is ill-posed. To seek a set of stable approximate solutions to the equation, a filter operator $g \lambda(s)$ is introduced as follows:

$$
g \lambda\left(s_{i}\right)=\frac{\lambda+s_{i}^{\sigma}}{s_{i}^{\sigma}}, \quad \sigma \geq 1,
$$

where $\lambda$ is the regularization parameter. The operator $g \lambda(s)$ is a modified operator including the traditional Tikhonov regularization operator. Therefore, equation (9) can be written as

$$
[H]^{+}=\sum_{i=1}^{m} \frac{\lambda+s_{i}^{\sigma}}{s_{i}^{\sigma}} \frac{1}{s_{i}} u_{i}^{T} v_{i} .
$$

With the increase of $\sigma$, the convergence order of the relative error of the regularization solution increases with it. From equation (10), it is found that the regularization parameter $\lambda$ plays an important role in the final solution. When the selected regularization parameter is very large, the load cannot be well identified; when the selected regularization parameter is small, the regularization solution of load identification will be unstable and cannot reasonably approximate the load identified. Therefore, a reasonable selection of regularization parameter is the key to the success of regularization solution. At present, the most used method for selecting regularization parameter is the $L$-curve criterion [23]. However, the $L$-curve is sometimes too smooth to find the $\lambda$ value corresponding to the maximum point of the bending derivative on the curve. Therefore, this paper used the Generalized Cross Validation (GCV) criterion to select the optimal regularization parameter. The GCV function is expressed as [24]

$$
G=\frac{\left\|[H]^{*}\left[S_{F F}\right]_{\mathrm{reg}}[H]^{T}-[\theta]\right\|_{2}^{2}}{\left(\operatorname{trace}\left(1-H H^{\mathrm{reg}}\right)\right)^{2}},
$$

where $H^{\text {reg }}=\left(H^{T} H+\lambda I\right)^{-1} H^{T}$ and satisfies $\left[S_{F F}\right]_{\text {reg }}=$ $\left[H^{\text {reg }}\right]^{*}\left[S_{Y Y}\right]\left[H^{\text {reg }}\right]^{T}$. When the GCV function takes the minimum value, the corresponding $\lambda$ value is the optimal regularization parameter.

\section{Single Pick Cutting Hard Rock Test}

3.1. Test Device. The test used the single pick test equipment of the National Engineering Laboratory of Coal Mining Machinery of China, as shown in Figure 1. The test equipment can simulate the process of the pick rock breaking, can test the cutting force, the wear state and the rock breaking rate produced by a single pick, and can change the cutting angle, cutting depth, and cutting speed of the single pick relative to the cutting object to obtain test processing such as three-direction cutting force, which provides fundamental test support for the design of pick and the cutting system. The test equipment is connected to an octagonal ring dynamometer and a multichannel data acquisition system, which can monitor and collect the threedirection force in the cutting process in real time. The sampling frequency is up to $20 \mathrm{kHz}$. The pick can be rotated in the direction of the arrow and moved back and forth. The rock can be moved left and right. The rotation speed of the pick determines the cutting speed. The installation angle of the pick relative to the pick seat determines the cutting angle. The left-and-right movement of the rock determines the cutting depth. The back-and-forth movement of the pick determines the cutting space. The relative movement of the pick and the rock completes the cutting process. To explore the applicability of the above-mentioned load identification method, this paper added a vibration acceleration test system to the single pick cutting test equipment. The system consists of KGS18 mine three-direction vibration 



(a)

(b)

FiguRE 1: Schematic drawing of single pick cutting rock test equipment. (a) Front view. (b) Vertical view. 1: base fixture; 2: stone moving device; 3: stone clamping device; 4: stone; 5: test control system; 6: test pick; 7: cutting force sensor; 8: cutting driving device; 9: tool holder moving device; 10: gearbox; 11: coupling; 12: driving belt; 13: driving motor; 14: cutting force collection device; 15: tool holder driving motor; 16: coupling; 17: driving screw; 18: dust removal system; 19: distribution cabinet; 20: hydraulic pump station; 21: dust suppression pump station; 22: locking handle; 23: tool holder moving rail; 24: protection assembly; 25: image acquisition system; 26: stone moving rail; 27: stone table driving motor; 28: coupling; 29: driving screw; 30: coupling; 31; stone table driving screw.

acceleration sensor and YHZ18 mine vibration monitoring analyzer.

The installation position of the test sensor is shown in Figure 2. The sensor layout method can be found in [25]. After collecting the vibration acceleration value, the threedirection force is obtained of the pick cutting by adopting the inverse pseudoexcitation method and the modified regularization method, which is compared with the force measured by the test equipment force measurement system to verify the accuracy of the method.

3.2. Test Object. The test object uses an alloy steel pick, whose material is $35 \mathrm{CrMnSiA}$ high-strength steel, the Vickers microhardness is $862 \mathrm{HV}$, the external elongation is $80 \mathrm{~mm}$, the pick shank diameter is $38 \mathrm{~mm}$, the pick tip diameter is $25 \mathrm{~mm}$, the edge diameter is $60 \mathrm{~mm}$, and the pick tip angle is $80^{\circ}$. The cutting object uses special hard rock with a size of $1200 \mathrm{~mm} \times 800 \mathrm{~mm} \times 600 \mathrm{~mm}$. The basic mechanical characteristics of the hard rock are obtained by performing uniaxial compressive strength tests and Brazilian tensile strength tests as shown in Table 1 . The cutting test adopts orthogonal test method. The test variables are the cutting depth, the cutting speed, the cutting angle, and the cutting spacing. The test

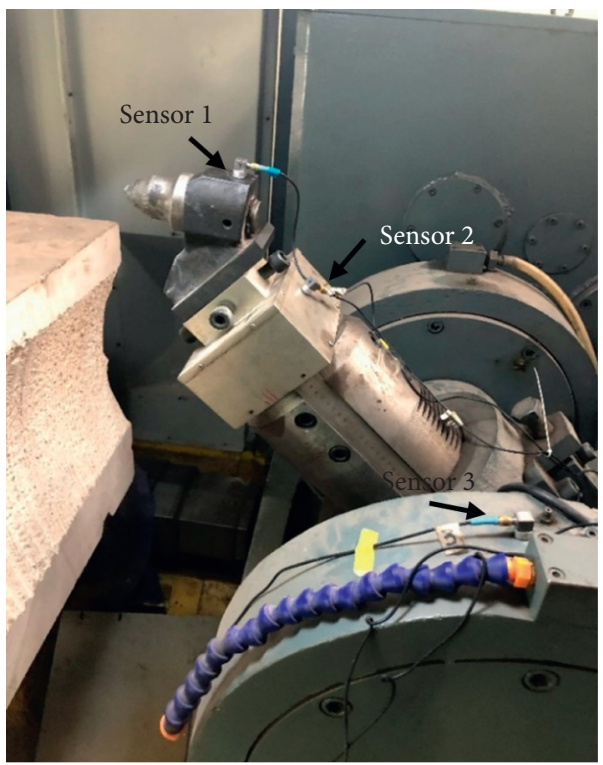

FiguRE 2: Sensor layout.

conditions are shown in Table 2. Each test condition is repeated 3 times, the test temperature is $19^{\circ} \mathrm{C}$, the sampling frequency of the dynamometer is $500 \mathrm{~Hz}$, and the 
TABLE 1: Mechanical characteristics of rock. \begin{tabular}{llllll}
\hline Rock type & $\gamma\left(\mathrm{kg} / \mathrm{m}^{3}\right)$ & UCS $(\mathrm{MPa})$ & $\mathrm{BTS}(\mathrm{MPa})$ & $E(\mathrm{GPa})$ & $v$
\end{tabular}

\begin{tabular}{llllll}
\hline Sandstone & 2340 & 61.7 & 4 & 21 & 0.26
\end{tabular}

UCS is uniaxial compressive strength; BTS is Brazilian tensile strength; $\gamma$ is density; $E$ is Young's modulus; $v$ is Poisson's ratio.

TABLe 2: Test conditions.

\begin{tabular}{lcccc}
\hline Test number & $\begin{array}{c}\text { Cutting } \\
\text { depth }(\mathrm{mm})\end{array}$ & $\begin{array}{c}\text { Cutting } \\
\text { speed } \\
(\mathrm{m} / \mathrm{s})\end{array}$ & $\begin{array}{c}\text { Cutting } \\
\text { angle }\left(^{\circ}\right)\end{array}$ & $\begin{array}{c}\text { Cutting } \\
\text { spacing }(\mathrm{mm})\end{array}$ \\
\hline 1 & 2 & 1.5 & 45 & 10 \\
2 & 2 & 2 & 49 & 20 \\
3 & 2 & 2.5 & 52 & 30 \\
4 & 3 & 1.5 & 49 & 30 \\
5 & 3 & 2 & 52 & 10 \\
6 & 3 & 2.5 & 45 & 20 \\
7 & 4 & 1.5 & 52 & 20 \\
8 & 4 & 2 & 45 & 30 \\
9 & 4 & 2.5 & 49 & 10 \\
\hline
\end{tabular}

sampling frequency of the vibration acceleration collector is $10240 \mathrm{~Hz}$.

\section{Results and Discussion}

To validate the applicability of the load identification method, the data in this paper is not subject to data processing. Taking test number 5 as an example, the time domain and frequency domain curves of the load measured by the dynamometer with the cutting time are shown in Figure 3. The vibration time domain signal and frequency domain curve of measuring point 1 under the same test condition are shown in Figure 4. The signal taken is perpendicular to the cutting plane direction. As can be seen from Figures 3(a) and 4(a), the load and vibration amplitude are fluctuating widely with cutting time, indicating that the breaking of the rock by the pick is a nonuniform stable process in the cutting process. This is due to the heterogeneity of the materials during the formation of the rock and also due to the low power of the cutting motor used in the test. Therefore, the design of the cutting system should fully consider the impact of the peak load rather than the average load on the power of the cutting motor. As can be seen from the frequency domain responses in Figures 3(b) and 4(b), one of the characteristic frequencies of the cutting force is $3.62 \mathrm{~Hz}$ and that of the cutting vibration is $112.24 \mathrm{~Hz}$. The cutting vibration frequency is 31 times the cutting force frequency, indicating that the characteristic value of the vibration signal contains the characteristic value of the cutting force, which is exactly an integer multiple of it. Therefore, the feasibility of the test can be determined and it further shows that the mechanical vibration and load have a certain correlation in the cutting process of a single pick.

Figure 5 shows the variation trends of the load obtained by the dynamometer and the identified load by the method in this paper with the cutting depth, the cutting speed, the cutting angle, and the cutting spacing. As can be seen from Figure 5(a), the loads increase rapidly but nonlinearly with the increase of cutting depth. The influence of the cutting depth on the cutting system is greater than the other three factors. Therefore, when the cutting object is hard rock, the cutting depth should be as small as possible while considering the cutting efficiency.

Figure 5(b) shows the variation in the collected load and the identified load with the cutting speed. As can be seen from the figure, the loads decrease slightly with the increase of cutting speed, indicating that the inertial force plays a certain role in it. Figure 5(c) shows the variation in the loads with the cutting angle. As can be seen from the figure, the loads first decrease with increase in the cutting angle up to $49^{\circ}$ and then start increasing with increase in the cutting angle. Therefore, there is an optimal cutting angle, which can produce a large rock breaking force. This is consistent with the conclusion of [3]. Figure 5(d) shows the variation with the loads with the cutting spacing. The correlation coefficient between the loads and the cutting spacing is small and no general law has been found. This may be due to the larger value of the cutting spacing in the test and there is no coincident cutting area. Figure 6 shows the trace of the single pick cutting rock interface. It can be seen that the traces are selected as the cut spacing is too large. The cutting tracks do not cross during the cutting process. However, in the actual tunneling or coal mining process, they will cause the relative change of the load because of the large number of picks and the different arrangement of the picks. Therefore, multipick cutting tests are needed to further determine the relationship between the cutting spacing and the cutting load.

As can also be seen from Figure 5, no matter what the parameters are, the change trend of the cutting load identified by the load identification method established in this paper and the actual measured load is consistent. The differences between the real loads and identified loads could also be seen in Figure 5. The maximum difference is $1.27 \mathrm{kN}^{2} / \mathrm{Hz}$, which is about $17.35 \%$ between real load and identified load, as shown in Figure 5(b), when the cutting speed is $1.5 \mathrm{~m} / \mathrm{s}$. This may be due to the relatively independent relationship between load and speed, resulting in the vibration signal having other unknown energy components. The frequency spectrum of vibration signal is processed under the test conditions, as shown in Figure 4(b). In addition to the system's natural characteristic frequency, it also includes a larger characteristic component, and the energy value of this component increases with the increase of cutting speed. The method described in this paper uses the amplitude of the frequency response function feature vector. Therefore, the increase of this energy component would have a certain impact on load identification [26-28]. In summary, within a certain range of motion speed, the load identification method has proved to have a high identification accuracy in this paper.

For comparison with the load identification method before improvement, with the regularization method which does not introduce a filter operator $g \lambda(s)$, the error quantization index is defined:

$$
\text { Error }=\frac{\left\|S_{1}-S_{2}\right\|}{\left\|S_{2}\right\|} \times 100 \%,
$$




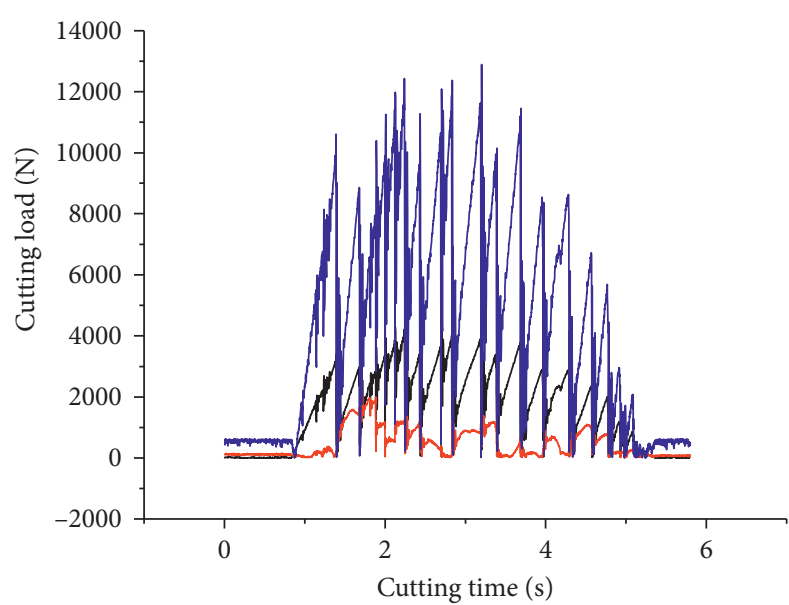

Cutting time (s)

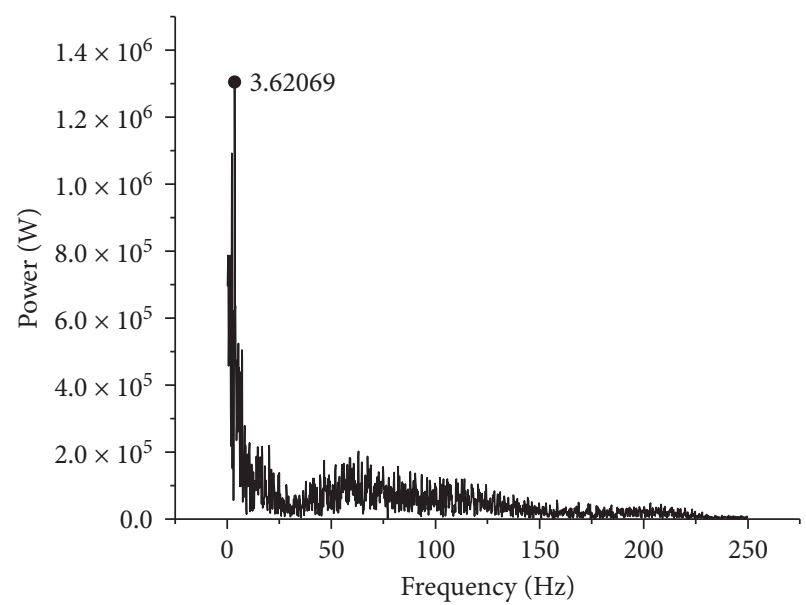

- Tangential force

_ Lateral force

- Normal force

(a)

(b)

Figure 3: Time domain (a) and frequency domain (b) of cutting load.

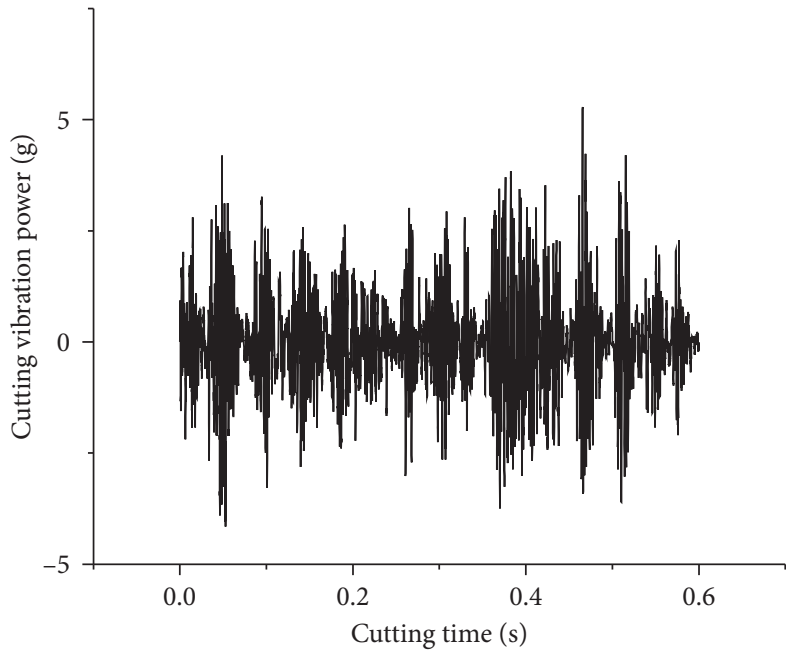

(a)

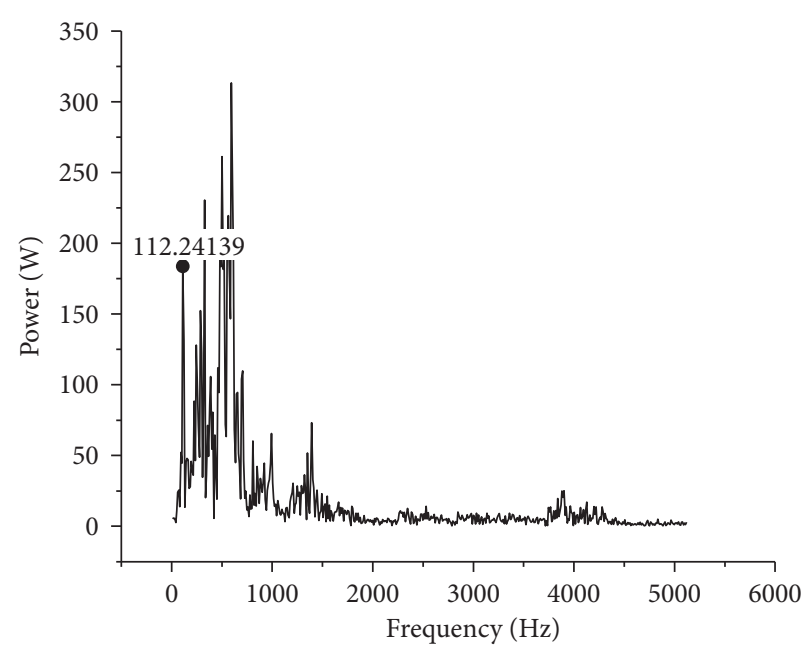

(b)

Figure 4: Cutting vibration time domain (a) and frequency domain (b).

where $S_{1}$ represents the root mean square of the amplitude of the identified load power spectrum; $S_{2}$ represents the root mean square of the real load power spectrum amplitude.

The identification errors are summarized in Table 3.

The improved regularization method in all three measurement points can reduce the load identification error, and the modified reduction error is up to $1.98 \%$. It can also be seen from Table 3 that the farther the sensor is from the cutting surface, the greater the load identification error is. The reason is that the components other than the cutting energy contained in the vibration signal have a greater impact on the load. Therefore, the problem of load identification can be meaningful if it is discussed within a certain range. However, the improved load identification method in 

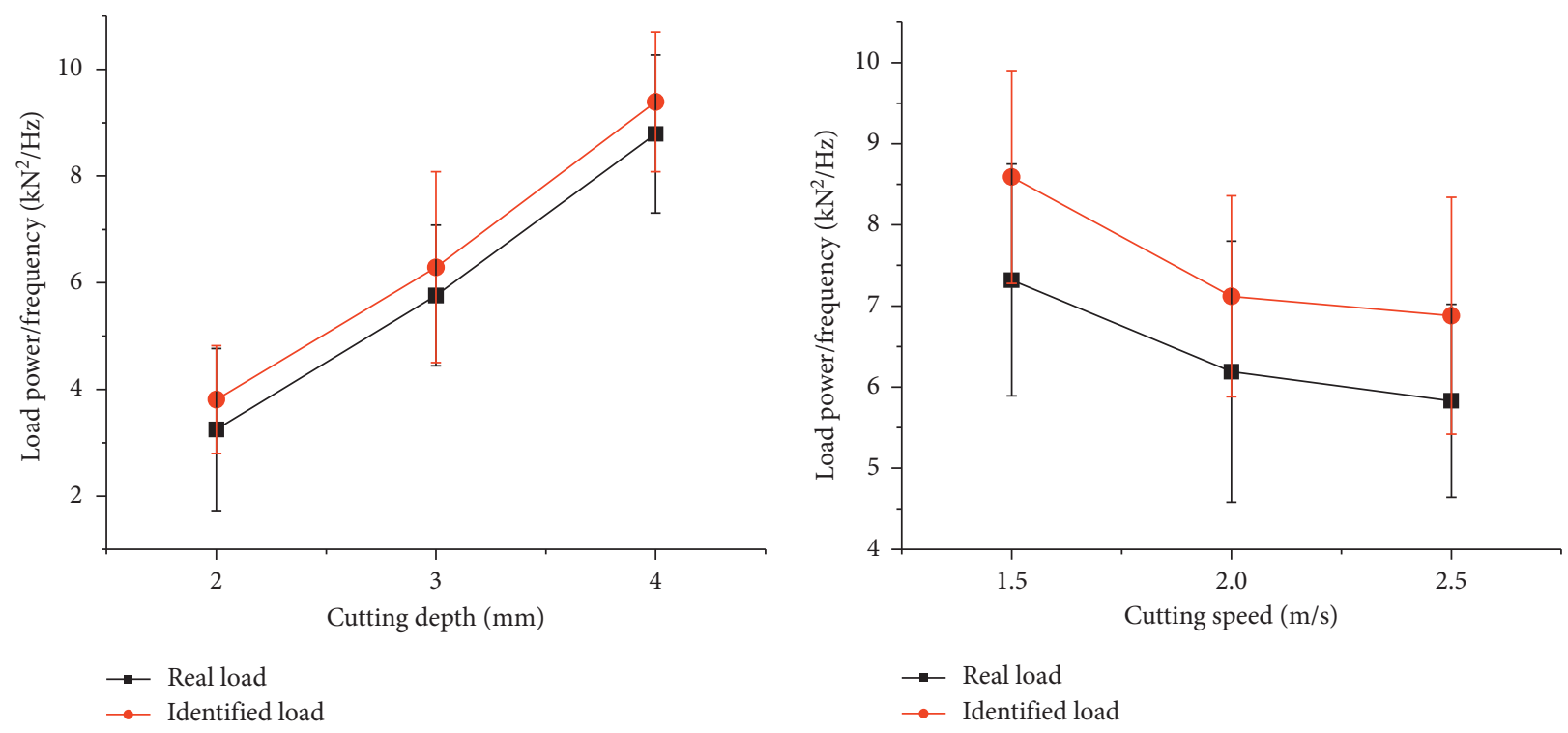

(a)

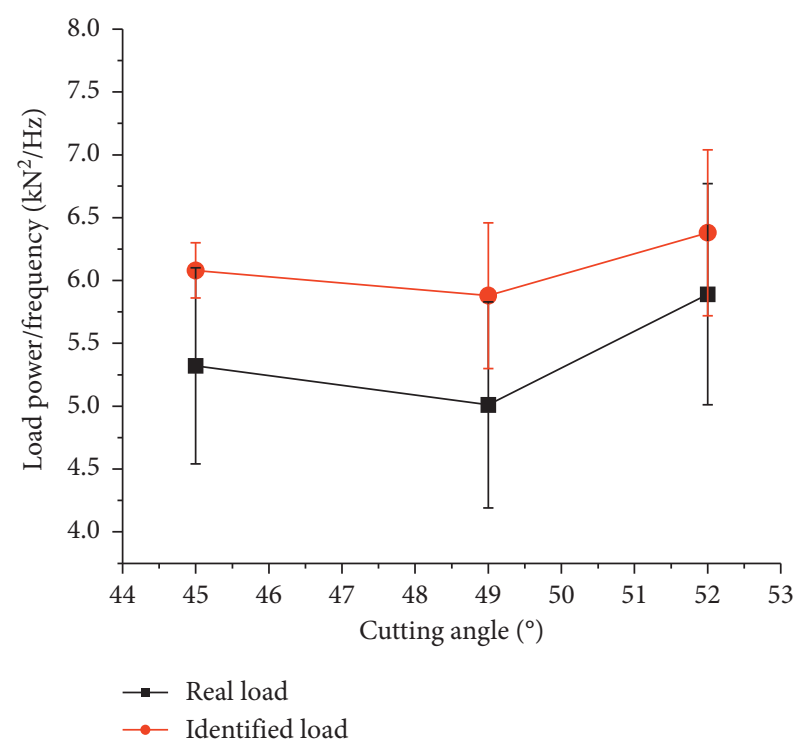

(c)

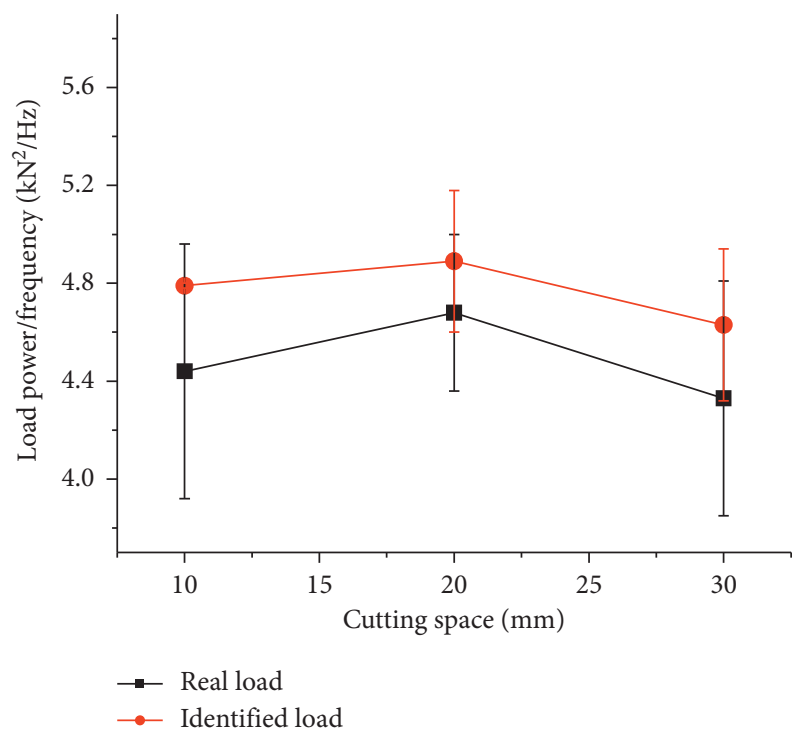

(d)

Figure 5: Comparison curves of real load and identified load: (a) cutting depth, (b) cutting speed, (c) cutting angle, and (d) cutting space.

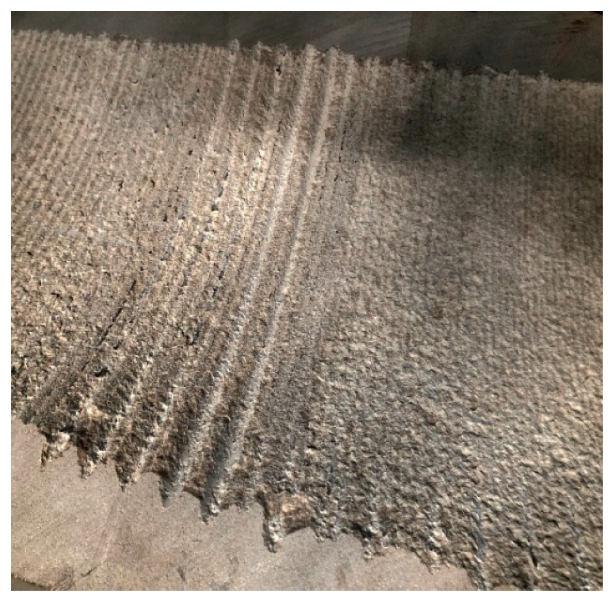

FIgURE 6: Single pick cutting section. 
TABLE 3: Recognition error of three measuring point loads by traditional method and this paper's method.

\begin{tabular}{lccc}
\hline Method & & Error values (\%) & \\
& Measuring point 1 & Measuring point 2 & Measuring point 3 \\
\hline Before improvement & 12.18 & 14.02 & 17.63 \\
After improvement & 10.20 & 13.11 & 17.35 \\
\hline
\end{tabular}

this paper can modify the inverse distortion problem of the frequency response function around the natural frequency to a certain extent.

\section{Conclusions}

(1) This paper improves the traditional regularization method by introducing a filter operator. The test of cutting rock by a pick verifies the method. The maximum difference between the identified load and the real load is $17.35 \%$. The cutting speed has the greatest influence on the identified load accuracy.

(2) Compared with the unimproved regularization method, the improved load identification method can reduce the identification error up to $1.98 \%$. The closer the sensor is to the load acting position, the better the improvement effect is.

(3) The phenomenon of energy concentration in the frequency spectrum of the pick cutting vibration signal with the increase of the speed should be further studied and analyzed, as it provides another research topic for improving the accuracy of load identification.

\section{Data Availability}

The data used to support the findings of this study are available from the corresponding author upon request.

\section{Disclosure}

This paper has been presented as preprint in Research Square (DOI: 10.21203/rs.3.rs-57473/v1).

\section{Conflicts of Interest}

The authors declare that there are no conflicts of interest regarding the publication of this paper.

\section{Authors' Contributions}

Conceptualization was performed by Lei Dong and Ruimin Shi; methodology was provided y Bukang Wang and Junyuan Wang; validation was done by Siyu Zhai; formal analysis was carried out by Ruimin Shi and Lei Dong; investigation was conducted by Lei Dong; resources were provided by Bukang Wang; data curation was done by Liang Dong; original draft was written by Lei Dong and Ruimin Shi; review and editing were carried out by Lei Dong; visualization and supervision were performed by Ruimin Shi; project administration was conducted by Bukang Wang and Junyuan Wang; and funding acquisition was carried out by Lei Dong and Ruimin Shi.

\section{Acknowledgments}

The authors are grateful to the National Natural Science Foundation of China (51875152) and the Natural Science Foundation of Shanxi Province (201901D211200).

\section{References}

[1] P. Wang, G. L. Yang, and H. Xiao, "Dynamic load identification theoretical summary and the application on mining machinery," Applied Mechanics and Materials, vol. 330, pp. 811-814, 2013.

[2] C. Dogruoz and N. Bolukbasi, "Effect of cutting tool blunting on the performances of various mechanical excavators used in low- and medium-strength rocks," Bulletin of Engineering Geology and the Environment, vol. 73, no. 3, pp. 781-789, 2014.

[3] D. Yang, J. Li, L. Wang, K. Gao, Y. Tang, and Y. Wang, "Experimental and theoretical design for decreasing wear in conical picks in rotation-drilling cutting process," International Journal of Advanced Manufacturing Technology, vol. 77, no. 9-12, pp. 1571-1579, 2015.

[4] X. H. He, X. G. Hua, Z. Q. Chen, and F. L. Huang, "EMDbased random decrement technique for modal parameter identification of an existing railway bridge," Engineering Structures, vol. 33, no. 4, pp. 1348-1356, 2011.

[5] A. D. Raath and C. C. V. Waveren, "A time domain approach to load reconstruction for durability testing," Engineering Failure Analysis, vol. 5, no. 2, pp. 113-119, 1998.

[6] J. Liu, X. Meng, C. Jiang, X. Han, and D. Zhang, "Timedomain Galerkin method for dynamic load identification," International Journal for Numerical Methods in Engineering, vol. 105, no. 8, pp. 620-640, 2016.

[7] X. Wu, X. Han, L. Liu, and B. Qi, "A load identification algorithm of frequency domain filtering under current underdetermined separation," IEEE Access, vol. 6, pp. 37094-37107, 2018.

[8] M.-H. Lee and Y.-W. Liu, "Input load identification of nonlinear tower structural system using intelligent inverse estimation algorithm," Procedia Engineering, vol. 79, pp. 540-549, 2014.

[9] X. Li and Z. Deng, "Identification of dynamic loads based on second-order Taylor-series expansion method," Shock and Vibration, vol. 2016, Article ID 6461427, 9 pages, 2016.

[10] L. Zhi, P. Yu, Q.-S. Li, B. Chen, and M. Fang, "Identification of wind loads on super-tall buildings by Kalman filter," Computers \& Structures, vol. 208, pp. 105-117, 2018.

[11] L. Wang, X. Wang, and X. Li, "Inverse system method for dynamic loads identification via noisy measured dynamic responses," Engineering Computations, vol. 33, no. 4, pp. 1070-1094, 2016.

[12] J. Zhou, L. Dong, W. Guan, and J. Yan, "Impact load identification of nonlinear structures using deep recurrent neural network," Mechanical Systems and Signal Processing, vol. 133, Article ID 106292, 2019.

[13] M. A. Hassan, A. R. Bushroa, and R. Mahmoodian, "Identification of critical load for scratch adhesion strength of 
nitride-based thin films using wavelet analysis and a proposed analytical model," Surface and Coatings Technology, vol. 277, pp. 216-221, 2015.

[14] S. S. Patel, A. Chourasia, S. K. Panigrahi, S. K. Bhattacharyya, and J. Parashar, "A study on efficacy of wavelet transform for damage identification in reinforced concrete buildings," Journal of Vibration Engineering \& Technologies, vol. 6, no. 2, pp. 127-138, 2018.

[15] L. Wei and J. Zhang, "Parameter identification of comprehensive load modeling based on improved genetic algorithm," IOP Conference Series, Earth and Environmental Science, vol. 170, Article ID 042122, 2018.

[16] J. Treetrong, J. K. Sinha, F. S. Gu, and A. Ball, "An investigation of genetic algorithm based motor parameter estimation for condition monitoring," Journal of Vibration Engineering \& Technology, vol. 2, no. 2, pp. 97-115, 2014.

[17] F. Ren, A.-Q. Shi, and Z.-J. Yang, "Research on load identification of mine hoist based on improved support vector machine," Transactions of the Canadian Society for $\mathrm{Me}$ chanical Engineering, vol. 42, no. 3, pp. 201-210, 2018.

[18] H. G. Choi, A. N. Thite, and D. J. Thompson, "A threshold for the use of Tikhonov regularization in inverse force determination," Applied Acoustics, vol. 67, no. 7, pp. 700-719, 2006.

[19] H. G. Choi, A. N. Thite, and D. J. Thompson, "Comparison of methods for parameter selection in Tikhonov regularization with application to inverse force determination," Journal of Sound and Vibration, vol. 304, no. 3-5, pp. 894-917, 2007.

[20] E. Jacquelin, A. Bennani, and P. Hamelin, "Force reconstruction: analysis and regularization of a deconvolution problem," Journal of Sound and Vibration, vol. 265, no. 1, pp. 81-107, 2003.

[21] X. Song, Y. Bai, Z. Cheng, X. Gu, L. Liu, and D. Liang, “A dynamic load identification method for nonlinear beam system," Journal of Vibration Engineering \& Technologies, vol. 31, no. 1, pp. 82-90, 2018.

[22] X. Song, H. Zhai, and D. Liang, "Dynamic load identification and displacement prediction based on FBG for a cantilever beam," Journal of Vibration Engineering \& Technologies, vol. 7, no. 2, pp. 131-137, 2019.

[23] P. C. Hansen, The L-Curve and its Use in the Numerical Treatment of Inverse Problems, IMM, Department of Mathematical Modelling, Technical University of Denmark, Kongens Lyngby,Denmark, 1999.

[24] Y. Mao, X. Guo, and Y. Zhao, "A state space force identification method based on Markov parameters precise computation and regularization technique," Journal of Sound and Vibration, vol. 329, no. 15, pp. 008-3019, 2010.

[25] F. Yang, F. Zhang, and Y. Pu, "Study on sensor placement for distributed load identification," Journal of Vibration Engineering \& Technologies, vol. 30, no. 3, pp. 403-412, 2017.

[26] Y. Zhang and A. Wang, "Research on the fault diagnosis method for rolling bearings based on improved VMD and automatic IMF acquisition," Shock and Vibration, vol. 2020, Article ID 6216903, 19 pages, 2020.

[27] J. Fu, F. Cai, Y. Guo, H. Liu, and W. Niu, "An improved VMD-based denoising method for time domain load signal combining wavelet with singular spectrum analysis," Mathematical Problems in Engineering, vol. 2020, Article ID 1485937, 14 pages, 2020.

[28] S. Maurya, V. Singh, and N. K. Verma, "Condition monitoring of machines using fused features from EMD-based local energy with DNN," IEEE Sensors Journal, vol. 20, no. 15, pp. 8316-8327, 2020. 